\title{
An evolutionary power spectrum model of fully nonstationary seismic ground motion
}

\author{
Ding Wang ${ }^{\mathrm{a}, *}$, Zenglei Fan $^{\mathrm{a}}$, Shengwang $\mathrm{Hao}^{\mathrm{a}, \mathrm{b}}$, Dahai Zhao

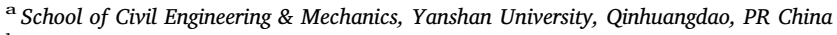 \\ ${ }^{\mathrm{b}}$ The State Key Laboratory of Nonlinear Mechanics, Institute of Mechanics, Chinese Academy of Science, Beijing, PR China
}

\section{A R T I C L E I N F O}

\section{Keywords:}

Evolutionary power spectral density

Seismic ground motion

Nonstationarity

Parameter identification

Generalized harmonic wavelets

\begin{abstract}
A B S T R A C T
A parametric evolutionary power spectrum model of fully nonstationary seismic ground motion is developed based on the evolutionary spectrum estimation method via generalized harmonic wavelets. The model consists of a frequency-domain energy distribution function and a series of normalized time-dependent envelop functions for different frequencies. The frequency-domain energy distribution function describes the spectral characteristics of the seismic ground motion. The nonstationarity is achieved by the normalized time-dependent envelope functions. For a specific seismic ground motion record, the evolutionary power spectral density (EPSD) is estimated via generalized harmonic wavelets. The parameters are identified by letting the model approximate to the estimated EPSD of the realistic ground motion. By using the spectral representation method, the proposed EPSD model can be used to synthesize the artificial seismic ground motion time histories for engineering purposes.
\end{abstract}

\section{Introduction}

Nonstationary characteristics of seismic ground motions have been of interest to seismologists and earthquake engineers for a long time. Generally, the realistic seismic ground motion exhibits two types of nonstationarities, namely temporal and spectral nonstationarities [1]. The temporal nonstationarity refers to the time variation of the intensity of the ground motion in the time domain and the spectral nonstationarity refers to the time variation of the energy distribution of the ground motion in the frequency domain. The physical factors causing the nonstationarity of the seismic ground motion are complex, including the onset and end of the earthquake fault rupture process, the seismic wave propagation through the random earth medium and the local site effect. As well known, both of the temporal and spectral nonstationarities of the earthquake ground motion may have significant effect on the response of nonlinear structure [2,3]. Hence, for the seismic response analysis of nonlinear structure, there has been a number of work regarding the modeling and the simulation of nonstationary seismic ground motion $[1,4-6]$.

Due to the complexity and randomness of the realistic earthquake time history, seismic ground motion is usually treated as stochastic process [7]. For engineering purposes, seismic ground motion is usually modeled as filtered white noise with amplitude modulation in time. Several amplitude envelope functions have been proposed to achieve the temporal nonstationarity of the filtered white noise model [8-11].
However, the amplitude-modulated filtered white noise model can not reflect the spectral nonstationarity of the realistic seismic ground motion. In 1965, Priestley defined the evolutionary power spectral density (EPSD) to describe the time variation of the frequency-domain energy distribution of the fully nonstationary stochastic process [12]. The EPSD was introduced to model the seismic ground motion by Liu in 1970 [13]. At present, several EPSD models of fully nonstationary seismic ground motions have been proposed [2,14-18]. For simulating stochastic process samples by power spectra, the spectral representation method, which was first presented by Shinozuka [19,20], has been developed in recent decades [21-23]. Applying the spectral representation method, the EPSD models are available to synthesize the artificial nonstationary and spectrum-compatible ground motion processes for structural nonlinear response analysis and seismic reliability evaluation.

Modeling of the EPSD of nonstationary seismic ground motion is based on the EPSD estimation of realistic ground motion record. With the development of the time-frequency analysis technology, especially the wavelet transforms, several EPSD estimation methods have been presented [24-26]. In 1994, Spanos and Failla proposed a general method to estimate the EPSD of nonstationary stochastic process using wavelets. Further, the harmonic wavelets (HW) and the generalized harmonic wavelets (GHW), which were both proposed by Newland $[27,28]$, have been applied in the EPSD estimation of the nonstationary stochastic process [25]. The development of the wavelet-based EPSD

\footnotetext{
* Corresponding author.

E-mail addresses: wangding@ysu.edu.cn (D. Wang), fanzenglei1011@163.com (Z. Fan), hsw@ysu.edu.cn (S. Hao), zhaodh@ysu.edu.com (D. Zhao).
} 
estimation technology has provided a basis for the EPSD modeling of the nonstationary seismic ground motion.

In this article, an evolutionary power spectrum model of fully nonstationary seismic ground motion is proposed, including: a). Within the framework of evolutionary spectra estimation given by Spanos and Failla [24], an EPSD estimation formula for nonstationary process is derived based on the GHW. This EPSD estimation formula is expressed by the GHW coefficients. For a realistic ground motion record at station C00, the SMART-1 array, the GHW coefficients are computed and the corresponding EPSD is obtained. b). An parametric EPSD model of nonstationary seismic ground motion is proposed, consisting of a frequency-domain energy distribution function and a series of normalized time-dependent envelop functions for different frequency components. c). The parameter identification method is given. The parameters of the normalized time-dependent envelop functions should be respectively identified for different frequencies. In order to simplify the model, the parameters of the normalized envelop functions are treated as function of frequency. d). Based on the spectral representation method, the seismic ground motion acceleration samples are synthesized by using the proposed EPSD model.

\section{EPSD estimation via GHW}

As defined by Priestley [12], a zero mean nonstationary process $f(t)$ can be expressed as

$f(t)=\int_{-\infty}^{+\infty} A(\omega, t) \cdot e^{i \omega t} \cdot \mathrm{d} Z(\omega)$

in which $A(\omega, t)$ is a deterministic modulating function and $Z(\omega)$ is a spectral process with orthogonal increments. The evolutionary power spectral density of $f(t)$ is defined as

$S_{f f}(\omega, t)=|A(\omega, t)|^{2} \cdot S_{\overline{f f}}(\omega)$

in which $S_{f f}(\omega)$ is the power spectral density of the associated stationary process

$\bar{f}(t)=\int_{-\infty}^{+\infty} e^{i \omega t} \cdot \mathrm{d} Z(\omega)$

and satisfies

$E\left[|\mathrm{~d} Z(\omega)|^{2}\right]=S_{f f}(\omega) \cdot \mathrm{d} \omega$

In Eq. (4), $E[\cdot]$ represents the expectation operator. The EPSD reflects the time-varying frequency-domain energy distribution of the nonstationary stochastic process and is a powerful tool to describe the nonstationary properties of a realistic signal. For engineering applications, according to the algorithm in [22], the EPSD is available to simulate the fully nonstationary process by spectral representation by the following series as $N \rightarrow \infty$

$f_{0}(t)=\sqrt{2} \sum_{n=0}^{N-1}\left[2 S_{f f}\left(\omega_{n}, t\right) \cdot \Delta \omega\right]^{1 / 2} \cos \left(\omega_{n} \cdot t+\Phi_{n}\right)$

in which $f_{0}(t)$ is the simulated process, $\omega_{n}=(n-1) \cdot \Delta \omega$, $n=1,2, \ldots, N$, are the discrete frequencies and $\Phi_{n}, n=1,2, \ldots, N$, are the independent random phase angles uniformly distributed in the range of $[0,2 \pi]$.

Generally, the EPSD of a realistic process can be estimated via wavelets [24]. The normalized wavelet transform $W(a, b)$ of a function $X$ $(t)$ is defined as

$W(a, b)=\frac{1}{\sqrt{|a|}} \int_{-\infty}^{+\infty} X(t) \cdot w^{*}\left(\frac{t-b}{a}\right) \mathrm{d} t$

in which $w(t)$ is the mother wavelet function, $a$ is the scale parameter and $b$ is the time parameter. The asterisk denotes the complex conjugate. The kernel of wavelet transform (6) is the conjugation of the mother wavelet function with dilatation $a$ and translation $b$. Spanos and Failla derived that [24], for a given scale parameter $a_{j}$, the EPSD
$S_{f f}(\omega, t)$ of a realistic stochastic process $f(t)$ satisfied

$E\left[W\left(a_{j}, b\right)^{2}\right]=4 \pi^{2} a_{j} \int_{-\infty}^{+\infty}\left|F_{w}\left(\omega \cdot a_{j}\right)\right|^{2} \cdot S_{f f}(\omega, b) \cdot \mathrm{d} \omega$

in which $F_{w}(\omega)$ was the Fourier transform of the mother wavelet function $w(t)$, as

$F_{w}(\omega)=\frac{1}{2 \pi} \int_{-\infty}^{+\infty} w(t) \cdot e^{-i \omega t} \cdot \mathrm{d} t$

The EPSD, $S_{f f}(\omega, t)$, could be estimated by solving integral Eq. (7). In this article, the GHW are used in the EPSD estimation. The GHW function by time step $k /(n-m)$ is defined as

$w_{m, n}\left(t-\frac{k}{n-m}\right)=\frac{\exp \left\{2 \pi n i\left(t-\frac{k}{n-m}\right)\right\}-\exp \left\{2 \pi m i\left(t-\frac{k}{n-m}\right)\right\}}{2 \pi i(n-m)\left(t-\frac{k}{n-m}\right)}$

in which $m, n$ are the scale parameters of the GHW and $k$ must be an integer. The dimensions of $m, n$ are both Hz. The Fourier transform of Eq. (9) is [28]

$F_{w_{m, n}}(\omega)= \begin{cases}\frac{1}{2 \pi(n-m)} \exp \{-i \omega k /(n-m)\}, & 2 \pi m \leq \omega<2 \pi n \\ 0, & \text { elsewhere }\end{cases}$

Fig. 1a presents the Fourier spectrum of a single GHW of level $m, n$. The Fourier spectra of the GHW of different levels are shown in Fig. 1b. As derived by Newland [28], the GHW coefficients $c_{m, n, k}$ of a function $X$ $(t)$ are

$c_{m, n, k}=(n-m) \cdot \int_{-\infty}^{+\infty} X(t) \cdot w_{m, n}^{*}\left(t-\frac{k}{n-m}\right) \mathrm{d} t$

$\widetilde{c}_{m, n, k}=(n-m) \cdot \int_{-\infty}^{+\infty} X(t) \cdot w_{m, n}\left(t-\frac{k}{n-m}\right) \mathrm{d} t$

and $X(t)$ can be expressed as

a).

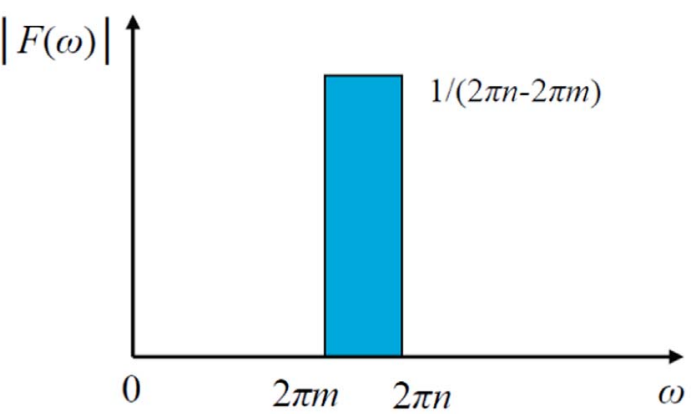

b).

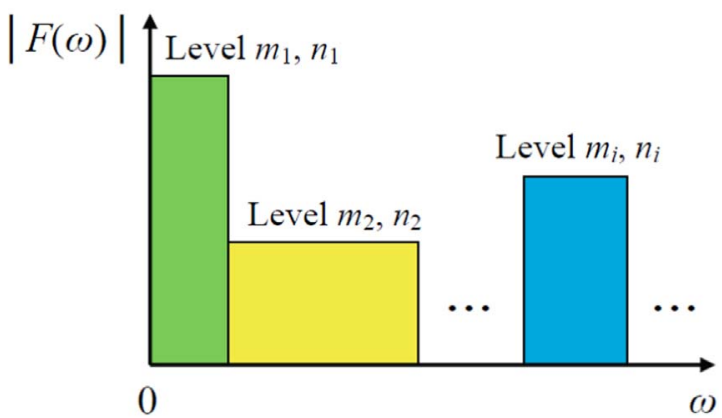

Fig. 1. Fourier amplitude spectra of a). a single GHW of level $m, n$ and b). of the GHW of different levels. 
$X(t)=\sum_{m, n} \sum_{k=-\infty}^{+\infty}\left\{c_{m, n, k} \cdot w_{m, n}\left(t-\frac{k}{n-m}\right)+\tilde{c}_{m, n, k} \cdot w_{m, n}^{*}\left(t-\frac{k}{n-m}\right)\right\}$

The GHW transform $W_{m, n, k}$ of a function $X(t)$ is defined as

$W_{m, n, k}=\int_{-\infty}^{+\infty} X(t) \cdot w_{m, n}^{*}\left(t-\frac{k}{n-m}\right) \mathrm{d} t$

For the kernel of the GHW transform (14), the time translation is $k /(n-m)$ and the dilatation is controlled by the scale parameters $m, n$ instead of $a$. Comparing Eq. (14) with the general form of wavelet transform (6), it is obtained that $b$ and $a$ in Eq. (6) equal to $k /(n-m)$ and 1 respectively, and $m, n$ control the dilatation of the wavelet transform kernel. Hence, for the GHW transform, Eq. (7) is written as

$E\left[W_{m, n, k}^{2}\right]=4 \pi^{2} \int_{-\infty}^{+\infty}\left|F_{w_{m, n}}(\omega)\right|^{2} \cdot S_{f f}\left(\omega, \frac{k}{n-m}\right) \cdot \mathrm{d} \omega$

From Eqs. (11) and (14), we get

$W_{m, n, k}=\frac{c_{m, n, k}}{n-m}$

Further, taking Eqs. (10) and (16) into (15),

$E\left[c_{m, n, k}^{2}\right]=\int_{2 m \pi}^{2 n \pi} S_{f f}\left(\omega, \frac{k}{n-m}\right) \cdot \mathrm{d} \omega$

Eq. (17) can be expressed as

$\frac{E\left[c_{m, n, k}^{2}\right]}{2 \pi(n-m)}=\frac{\int_{2 m \pi}^{2 n \pi} S_{f f}\left(\omega, \frac{k}{n-m}\right) \cdot \mathrm{d} \omega}{2 \pi(n-m)}$

The right side of Eq. (18) is the mean value of $S_{f f}(\omega, t)$ in the frequency interval $(2 m \pi, 2 n \pi]$ at the time step $t=k /(n-m)$. Here we use the mean value

$\bar{S}_{f f}(\omega, t)=\frac{\int_{2 m \pi}^{2 n \pi} S_{f f}(\omega, t) \cdot \mathrm{d} \omega}{2 \pi(n-m)}$

as the estimated value of $S_{f f}(\omega, t)$. Thus, the EPSD of the stochastic process $f(t)$ in the frequency interval $(2 m \pi, 2 n \pi]$ at the time step $t=k /(n-m)$ can be estimated as

$\widetilde{S}_{f f}(\omega, t) \approx \bar{S}_{f f}(\omega, t)=\frac{E\left[c_{m, n, k}^{2}\right]}{2 \pi(n-m)}$

in which $\widetilde{S}_{f f}(\omega, t)$ denotes the estimated value of the EPSD of $f(t)$.

In this article, the GHW of identical bandwidth, which means for different levels the bandwidth, $\Delta=n-m$, is a constant, is used to estimate the EPSD. The Fourier spectra of these GHW are shown in Fig. 2. From Eq. (20), the EPSD estimation formula can be written as

$S_{f f}(\omega, t) \approx \bar{S}_{f f}(\omega, t)=\frac{E\left[c_{m, n, k}^{2}\right]}{2 \pi \Delta}$

It should be noted that the estimated EPSD obtained by Eq. (21) is the average of the realistic EPSD on the rectangular element

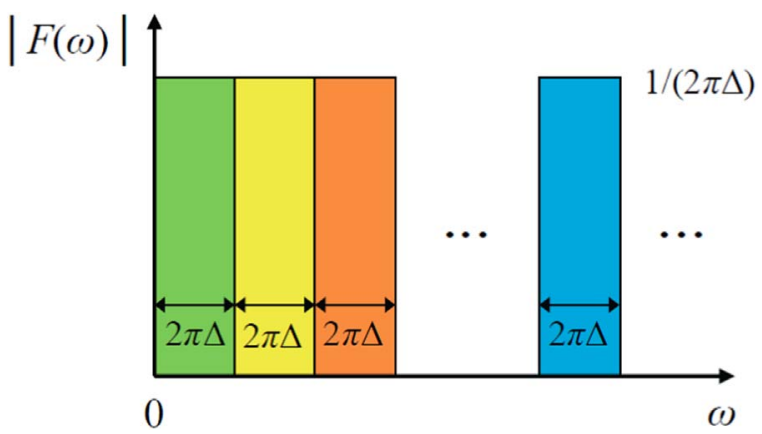

Fig. 2. Fourier amplitude spectra of the GHW of identical bandwidth.

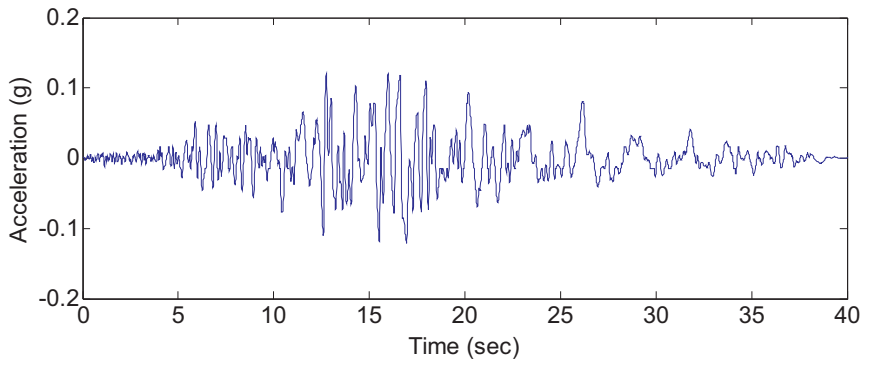

Fig. 3. Acceleration time history recorded at station C00, the SMART-1 array, Taiwan, during Event 45 in the E-W direction.

$[m, n) \times\left[\frac{k}{\Delta}, \frac{k+1}{\Delta}\right)$ in the frequency-time domain. The area of the rectangular element in the frequency-time domain is

$(n-m) \times\left(\frac{k+1}{\Delta}-\frac{k}{\Delta}\right)=\Delta \times \frac{1}{\Delta}=1$

which indicates that it is impossible to improve the frequency-domain estimation precision, $\Delta$, and the time-domain estimation precision, $1 / \Delta$, simultaneously. The little value of $\Delta$ will cause the high frequencydomain resolution but the low time-domain resolution, and vice versa.

Eq. (21) can be used to estimate the EPSD of realistic ground motion. Fig. 3 shows the seismic ground motion acceleration recorded at the center station C00 of the SMART-1 array, Taiwan, during Event 45 in the E-W direction. The estimated one-sided EPSD of this ground motion is shown in Fig. 4. As mentioned above, the estimated EPSD is the mean value of the realistic EPSD on the rectangular element in the frequency-time domain. Thus, in this paper, the bar graph is used to represent the estimated EPSD. In this example, the bandwidth, $\Delta$, is $1 \mathrm{~Hz}$ and, from Eq. (22), the corresponding time interval of the rectangular element is $1 \mathrm{~s}$. Fig. 5 presents the artificial seismic ground motion simulated using the estimated EPSD by Eq. (5). Intuitively, the nonstationary characteristics of the simulated ground motion is consistent with those of the realistic ground motion, which high frequency components are significant before $25 \mathrm{~s}$ but the low frequency component become remarkable after $25 \mathrm{~s}$.

\section{EPSD model of seismic ground motion}

As mentioned above, an appropriate nonstationary seismic ground motion model facilitates not only the stochastic response analysis of structural systems, but also their performance assessment allowing for the reliability evaluation of the system. For engineering purposes, the EPSD model of seismic ground motion should be able to describe the frequency-domain energy distribution and the intensity variations of different frequency components of the realistic ground motion record. In the field of seismology, the Fourier amplitude spectrum is usually used for modeling the earthquake source, path and site [29]. For a

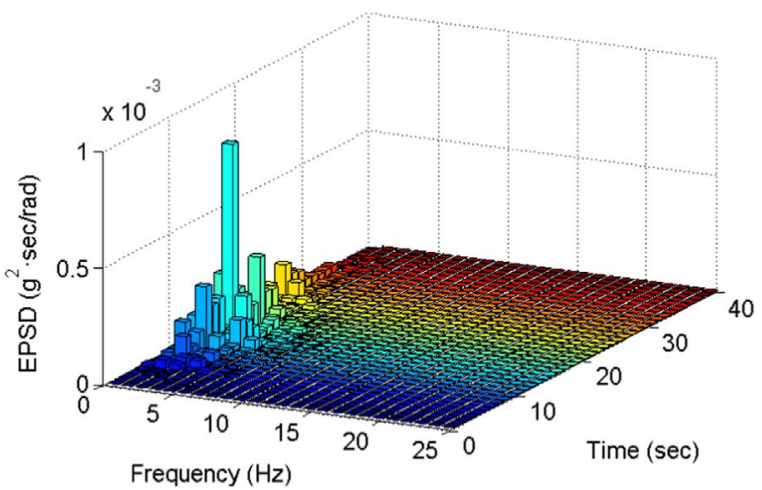

Fig. 4. Estimated EPSD of the earthquake ground motion record presented in Fig. 3. 


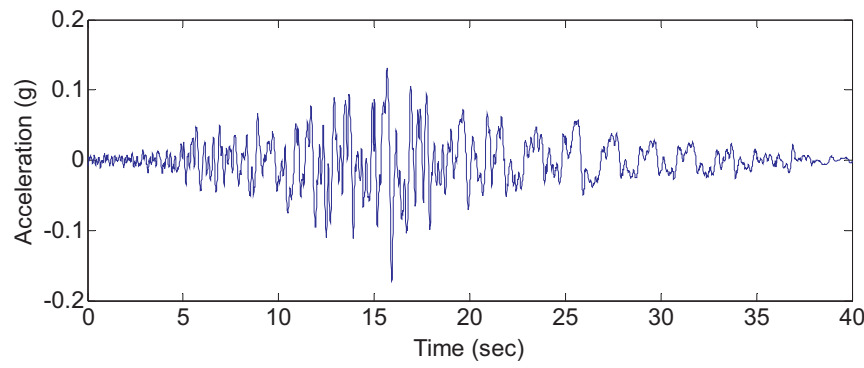

Fig. 5. Simulated ground motion acceleration using the estimated EPSD.

specific ground motion record, the power spectrum is proportional to the square of the Fourier amplitude spectrum [30]. In this article, the EPSD of the nonstationary seismic ground motion acceleration is expressed as

$S_{g}(\omega, t)=A_{g}^{2}(\omega, t)$

Physically, the spectral characteristics of the seismic ground motion are affected by earthquake source, path, site and instrument [29]. Here, the effects of the earthquake source and the local site on the ground motion spectrum are considered, so that $A_{g}(\omega, t)$ can be represented as

$A_{g}(\omega, t)=\omega^{2} \cdot A_{0} \cdot A_{s}(\omega) \cdot H(\omega) \cdot a(\omega, t)$

in which, $A_{0}$ is a constant named as amplitude parameter, $A_{s}(\omega)$ is the shape function of the seismic source displacement spectrum, $H(\omega)$ is the spectral transfer function of the local site, $a(\omega, t)$ is the normalized time-dependent envelope function of the frequency component of $\omega$, which satisfies the normalization condition

$\int_{0}^{+\infty} a^{2}(\omega, t) \cdot \mathrm{d} t=1$

The energy distribution function of the seismic ground motion can be derived by Eq. (23), as

$$
\begin{aligned}
E(\omega) & =\int_{0}^{+\infty} S_{g}(\omega, t) \cdot \mathrm{d} t \\
& =\omega^{4} \cdot A_{0}^{2} \cdot A_{s}^{2}(\omega) \cdot H^{2}(\omega) \cdot \int_{0}^{+\infty} a^{2}(\omega, t) \cdot \mathrm{d} t \\
& =\omega^{4} \cdot A_{0}^{2} \cdot A_{s}^{2}(\omega) \cdot H^{2}(\omega)
\end{aligned}
$$

Taking Eqs. (24) and (26) into (23), the EPSD model can be rewritten as

$S_{g}(\omega, t)=E(\omega) \cdot a^{2}(\omega, t)$

For the EPSD model presented as Eq. (27), $A_{0}, A_{s}(\omega)$ and $H(\omega)$ determine the energy distribution of the seismic ground motion in the frequency domain, and $a(\omega, t)$ determines the temporal and spectral nonstationarities of the seismic ground motion.

The most commonly used model of the seismic source spectrum is the $\omega$-square model, which is first presented by Aki [31]. Based on Aki's model, several empirical and semi-empirical seismic source spectrum models have been proposed for the modeling and simulation of the seismic ground motions [32-34]. Here, the shape function of the Atkinson and Boore's spectrum model is used to describe the effect of the seismic source on the ground motion spectral characteristics [32]. The shape function of the seismic source spectrum is

$A_{s}(\omega)=\left[\frac{1-\varepsilon}{1+\left(\frac{\omega}{2 \pi f_{a}}\right)^{2}}+\frac{\varepsilon}{1+\left(\frac{\omega}{2 \pi f_{b}}\right)^{2}}\right]$

in which $\varepsilon$ is the seismic moment ratio and $f_{a}, f_{b}$ are the corner frequencies.

The local site effect, which is determined by the soil condition of the site, is largely independent of the seismic source and path and is usually simplified as a filter. In this article, a single-degree-of-freedom system filter is used to describe the effect of the local site. The transfer function of this filter is

$H(\omega)=\sqrt{\frac{1+4 \xi_{g}^{2}\left(\frac{\omega}{\omega_{g}}\right)^{2}}{\left[1-\left(\frac{\omega}{\omega_{g}}\right)^{2}\right]^{2}+4 \xi_{g}^{2}\left(\frac{\omega}{\omega_{g}}\right)^{2}}}$

in which $\xi_{g}$ and $\omega_{g}$ are respectively the equivalent damping ratio and natural circular frequency of the site soil.

The normalized time-dependent envelope function, $a(\omega, t)$, describes the intensity variation of different frequency components, which reflects the temporal and spectral nonstationarities of the seismic ground motion. A variety of envelope function models have been proposed in the artificial seismic ground motion simulation. However, most of these models are applied to modify the intensity variation of the whole ground motion process instead of each frequency component. In this article, according to Amin and Ang's envelope function model [10], the normalized time-dependent envelope function of different frequency components is expressed as

$a(\omega, t)= \begin{cases}0 & 0<t \leq t_{0, \omega} \\ I_{\omega}\left(t / t_{1, \omega}\right)^{2} & t_{0, \omega}<t \leq t_{1, \omega} \\ I_{\omega} & t_{1, \omega}<t \leq t_{2, \omega} \\ I_{\omega} \cdot e^{-c_{\omega}\left(t-t_{2 . \omega}\right)} & t_{2, \omega}<t\end{cases}$

and the corresponding cumulative energy function is

$$
\begin{aligned}
h_{\omega}(t) & =\int_{0}^{t} a^{2}(\omega, \tau) \cdot \mathrm{d} \tau \\
& =I_{\omega}{ }^{2} \cdot \begin{cases}0 & 0<t \leq t_{0, \omega} \\
\frac{1}{5}\left(t-t_{0, \omega}\right)^{5} /\left(t_{1, \omega}-t_{0, \omega}\right)^{4} & t_{0, \omega}<t \leq t_{1, \omega} \\
t-\frac{4 \cdot t_{1, \omega}}{5}-\frac{t_{0, \omega}}{5} & t_{1, \omega}<t \leq t_{2, \omega} \\
\frac{5 \cdot t_{2, \omega}-4 \cdot t_{1, \omega}-t_{0, \omega}}{5}+\frac{1-e^{-2 c_{\omega}\left(t-t_{2, \omega}\right)}}{2 c_{\omega}} & t_{2, \omega}<t\end{cases}
\end{aligned}
$$

In Eqs. (30) and (31), $t_{0, \omega}$ is the starting time of the frequency component of $\omega$. The amplitude of this frequency component is increasing in a $t$-square form from $t_{0, \omega}$ to $t_{1, \omega}$, keeps steady from $t_{1, \omega}$ to $t_{2, \omega}$, and is decreasing in a exponential form after $t_{2, \omega} . c_{\omega}$ is the attenuation parameter of the exponentially decreasing stage. The peak value $I_{\omega}$ is not independent and can be determined by the normalization condition (25). When $t \rightarrow+\infty$, the cumulative energy of the frequency component of $\omega$ is

$$
\begin{aligned}
& \int_{0}^{+\infty} a^{2}(\omega, t) \cdot \mathrm{d} t \\
= & \lim _{t \rightarrow \infty}\left\{I_{\omega}^{2} \cdot\left(t_{2, \omega}-\frac{4 \cdot t_{1, \omega}}{5}-\frac{t_{0, \omega}}{5}+\frac{1-e^{-2 c_{\omega}\left(t-t_{2, \omega}\right)}}{2 c_{\omega}}\right)\right\} \\
= & I_{\omega}{ }^{2} \cdot\left(t_{2, \omega}-\frac{4}{5} t_{1, \omega}-\frac{1}{5} t_{0, \omega}+\frac{1}{2 c_{\omega}}\right)
\end{aligned}
$$

The normalization condition (25) indicates that the cumulative energy of each frequency component should equal to 1. For Eq. (32), the normalization condition requires

$I_{\omega}^{2} \cdot\left(t_{2, \omega}-\frac{4}{5} t_{1, \omega}-\frac{1}{5} t_{0, \omega}+\frac{1}{2 c_{\omega}}\right)=1$

$I_{\omega}$ can be derived from Eq. (33), as

$$
I_{\omega}=\frac{1}{\sqrt{t_{2, \omega}-\frac{4}{5} t_{1, \omega}-\frac{1}{5} t_{0, \omega}+\frac{1}{2 c_{\omega}}}}
$$




\section{Parameter identification}

Generally, for a specific seismic ground motion record, the parameter identification of the EPSD model includes three main steps, as

Step 1. Estimation of the EPSD of the ground motion record.

Step 2. Let the frequency-domain energy distribution function, $E(\omega)$, as shown by Eq. (26), approximate to the actual frequencydomain energy distribution of the realistic seismic ground motion record.

Step 3. For different frequency components, let the cumulative energy function, $h_{\omega}(t)$, as shown by Eq. (31), approximate to the actual cumulative energy curve of the realistic seismic ground motion.

The actual EPSD of the seismic ground motion record can be estimated by Eq. (20). Let $\widetilde{E}(\omega)$ and $\widetilde{h}_{\omega}(t)$ denote the actual frequencydomain energy distribution and the cumulative energy function. Both $\widetilde{E}(\omega)$ and $\widetilde{h}_{\omega}(t)$ can be calculated by the estimated EPSD of the realistic seismic ground motion, as

$\widetilde{E}(\omega)=\int_{0}^{+\infty} \widetilde{S}_{f f}(\omega, \tau) \cdot \mathrm{d} \tau$

$\widetilde{h}_{\omega}(t)=\frac{\int_{0}^{t} \widetilde{S}_{f f}(\omega, \tau) \cdot \mathrm{d} \tau}{\int_{0}^{+\infty} \widetilde{S}_{f f}(\omega, \tau) \cdot \mathrm{d} \tau}=\frac{\int_{0}^{t} \widetilde{S}_{f f}(\omega, \tau) \cdot \mathrm{d} \tau}{\widetilde{E}(\omega)}$

According to Eqs. (26) and (31), $E(\omega)$ includes the parameters $\alpha=\left(A_{0}, \quad \varepsilon, \quad f_{a}, \quad f_{b}, \quad \xi_{g}, \quad \omega_{g}\right)$, and for different frequencies, $h_{\omega}(t)$ includes the parameters $\beta_{\omega}=\left(t_{0, \omega}, \quad t_{1, \omega}, \quad t_{2, \omega}, \quad c_{\omega}\right)$. Let $\hat{\alpha}$ and $\hat{\beta}_{\omega}$ denote the identified values of $\alpha$ and $\beta_{\omega}$ respectively. The least-squares approximation is used during the parameter estimation. In the second step, the identified values of the parameters, $\hat{\alpha}$, minimize the integrated squared difference between $E(\omega)$ and $\widetilde{E}(\omega)$, i.e.

$\hat{\alpha}=\arg \min _{\alpha} \int_{0}^{\omega_{u}}[E(\omega)-\widetilde{E}(\omega)]^{2} \cdot \mathrm{d} \omega$

in which $\omega_{u}$ is the upper cutoff circular frequency. Similarly, in the third step, $\hat{\boldsymbol{\beta}}_{\omega}$ is obtained by minimizing the integrated squared difference between $h_{\omega}(t)$ and $\widetilde{h}_{\omega}(t)$, i.e.

$\hat{\beta}_{\omega}=\arg \min _{\beta_{\omega}} \int_{0}^{+\infty}\left[h_{\omega}(t)-\tilde{h}_{\omega}(t)\right]^{2} \cdot \mathrm{d} t$

For the seismic ground motion record shown in Fig. 3, the first line of Table 1 presents the identified values of the parameters in the frequency-domain energy distribution function. The comparison between the actual energy distribution of the ground motion record and the fitted model, shown in Fig. 6, indicates that Eq. (26) can well describe

Table 1

Identified parameter values of energy distribution function $E(\omega)$.

\begin{tabular}{lllllll}
\hline Station & $A_{0} / \mathrm{g} \cdot \mathrm{s}^{5 / 2} \cdot \mathrm{rad}^{2}$ & $\varepsilon$ & $f_{a} / \mathrm{Hz}$ & $f_{b} / \mathrm{Hz}$ & $\xi_{g}$ & $\omega_{g} / \mathrm{rad} / \mathrm{s}$ \\
\hline C00 & 0.294 & 0.046 & 0.051 & 0.134 & 0.623 & 9.406 \\
$\mathrm{I} 01$ & 0.294 & 0.026 & 0.057 & 0.074 & 0.812 & 9.136 \\
$\mathrm{I} 07$ & 0.291 & 0.046 & 0.053 & 0.131 & 0.617 & 7.962 \\
M01 & 0.209 & 0.025 & 0.065 & 0.071 & 0.693 & 8.133 \\
M07 & 0.275 & 0.050 & 0.047 & 0.175 & 0.486 & 10.150 \\
O01 & 0.280 & 0.048 & 0.046 & 0.145 & 0.646 & 10.288 \\
O02 & 0.268 & 0.044 & 0.044 & 0.133 & 0.466 & 19.675 \\
O04 & 0.306 & 0.042 & 0.055 & 0.111 & 0.697 & 8.753 \\
O06 & 0.268 & 0.028 & 0.061 & 0.075 & 0.818 & 7.6474 \\
O07 & 0.240 & 0.029 & 0.060 & 0.081 & 0.680 & 8.837 \\
O08 & 0.283 & 0.042 & 0.049 & 0.112 & 0.861 & 8.705 \\
O10 & 0.277 & 0.049 & 0.049 & 0.148 & 0.607 & 7.968 \\
O12 & 0.308 & 0.038 & 0.057 & 0.098 & 0.760 & 7.462 \\
Mean & 0.276 & 0.040 & 0.053 & 0.114 & 0.674 & 9.548 \\
Coefficient of & 0.098 & 0.236 & 0.118 & 0.290 & 0.178 & 0.332 \\
$\quad$ variation & & & & & & \\
\hline
\end{tabular}

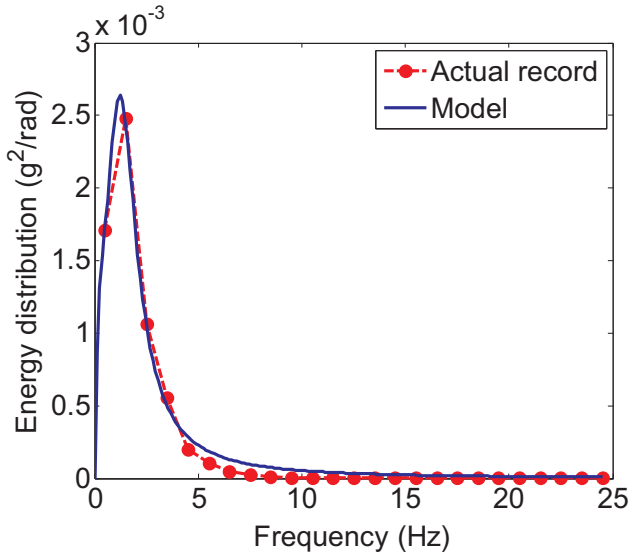

Fig. 6. Frequency-domain energy distribution of the actual ground motion record and fitted model.

Table 2

Identified parameter values of $a(\omega, t)$ in different frequency bands.

\begin{tabular}{|c|c|c|c|c|}
\hline Frequency band/Hz & $t_{0, \omega} / \mathrm{s}$ & $t_{1, \omega} / \mathrm{s}$ & $t_{2, \omega} / \mathrm{s}$ & $c_{\omega} / \mathrm{s}^{-1}$ \\
\hline $0-1$ & 2.941 & 9.673 & 26.134 & 0.158 \\
\hline $1-2$ & 0.000 & 12.598 & 14.986 & 0.128 \\
\hline $2-3$ & 0.000 & 16.046 & 16.047 & 0.173 \\
\hline $3-4$ & 0.000 & 12.289 & 12.289 & 0.155 \\
\hline $4-5$ & 0.000 & 7.766 & 17.759 & 0.206 \\
\hline $5-6$ & 0.000 & 12.922 & 16.494 & 0.180 \\
\hline $6-7$ & 0.000 & 10.245 & 15.633 & 0.154 \\
\hline $7-8$ & 0.000 & 5.937 & 15.516 & 0.109 \\
\hline $8-9$ & 0.000 & 7.229 & 17.378 & 0.230 \\
\hline $9-10$ & 0.000 & 5.357 & 19.982 & 0.227 \\
\hline 10-11 & 0.000 & 11.717 & 17.189 & 0.365 \\
\hline 11-12 & 0.000 & 12.194 & 17.606 & 0.284 \\
\hline $12-13$ & 0.000 & 7.805 & 18.494 & 0.191 \\
\hline $13-14$ & 0.000 & 7.457 & 18.542 & 0.249 \\
\hline 14-15 & 0.000 & 8.026 & 17.848 & 0.170 \\
\hline $15-16$ & 0.000 & 7.019 & 18.453 & 0.106 \\
\hline $16-17$ & 0.000 & 7.646 & 14.853 & 0.095 \\
\hline $17-18$ & 0.000 & 11.691 & 15.599 & 0.106 \\
\hline $18-19$ & 0.000 & 6.872 & 15.838 & 0.118 \\
\hline 19-20 & 0.000 & 3.200 & 17.967 & 0.050 \\
\hline $20-21$ & 0.000 & 5.745 & 20.164 & 0.089 \\
\hline $21-22$ & 0.000 & 10.855 & 18.537 & 0.097 \\
\hline $22-23$ & 0.000 & 3.396 & 16.404 & 0.054 \\
\hline $23-24$ & 0.000 & 0.001 & 20.597 & 0.058 \\
\hline 24-25 & 0.000 & 6.655 & 16.849 & 0.063 \\
\hline
\end{tabular}

the frequency-domain energy distribution of the realistic ground motion.

For different frequencies, the parameters in the normalized cumulative energy functions are estimated, shown in Table 2. Fig. 7 presents the comparison between the actual normalized cumulative energy curves of the ground motion record and the fitted models in different frequency bands. Fig. 8 presents the corresponding temporal variations of the energy of the realistic ground motion and those of the fitted model. The modeling results show that the normalized time-dependent envelope function (30) can describe the general tendency of the temporal energy variation of different frequency components. The EPSD model of the realistic ground motion is shown in Fig. 9. However, some details of the energy variation are lost during the identification. Fig. 10 shows the comparisons between the estimated ESPD of the realistic ground motion record and the fitted EPSD model from 8 to $17 \mathrm{~s}$. As a smoothed approximation of the actual EPSD, the fitted model can well describe the general spectral variation of the seismic ground motion record in the frequency domain, but can not reflect the peak and valley values of the actual EPSD quite exactly, such as during 14-15 s and $15-16 \mathrm{~s}$ shown in Fig. 10. 
a).

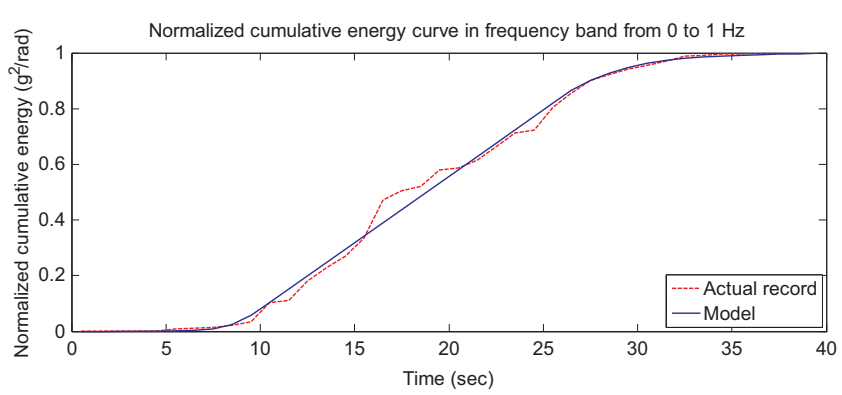

b).

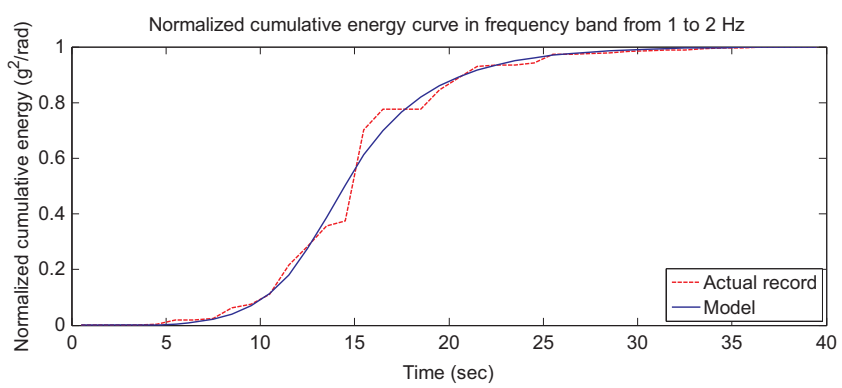

c).

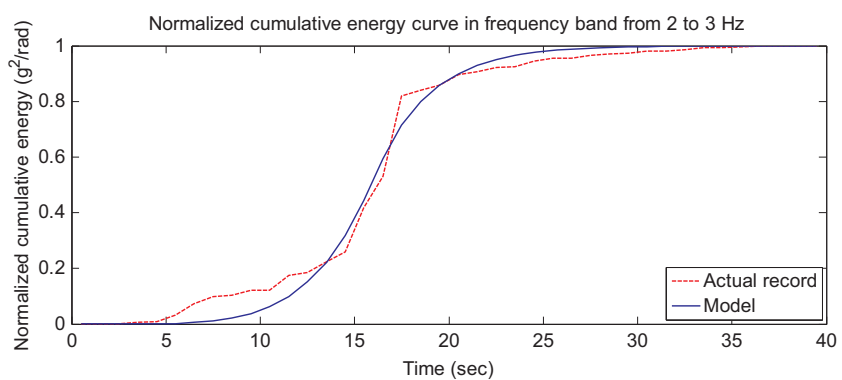

d).

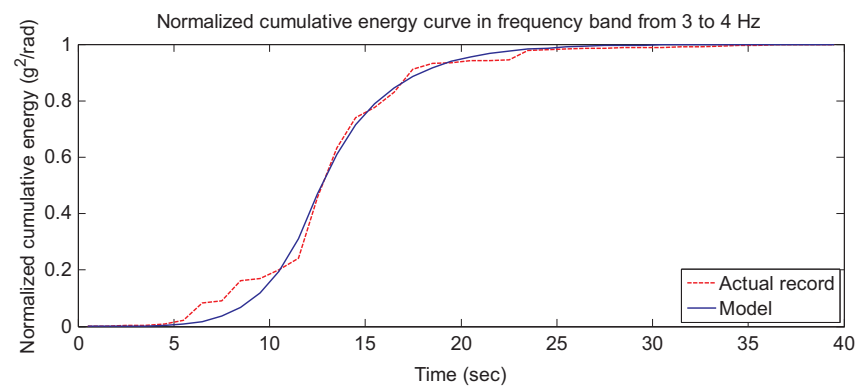

e).

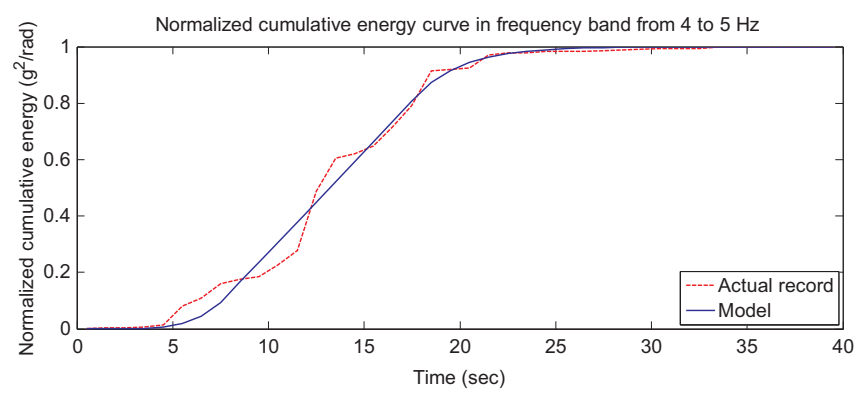

f).

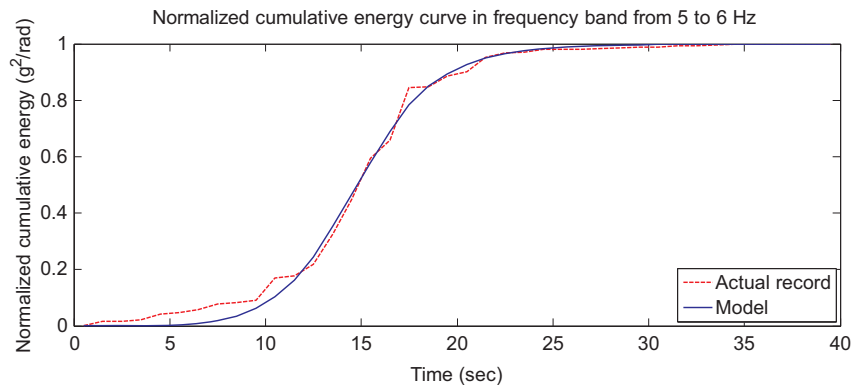

g).

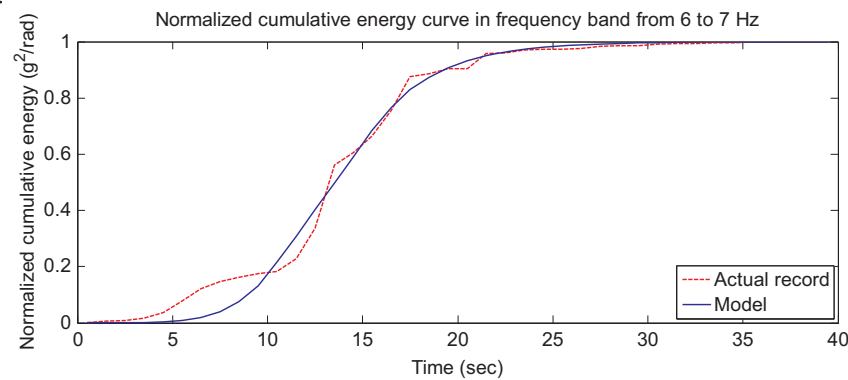

Fig. 7. Normalized cumulative energy curves of the actual ground motion record and fitted models in different frequency bands.

300 seismic ground motion samples are simulated by the spectral representation method using the fitted EPSD model. Fig. 11 presents one of the ground motion samples. The corresponding response spectra of these 300 samples are also computed. The comparison between the response spectrum of the realistic ground motion record and the mean response spectrum of the 300 samples are shown in Fig. 12.

It should be noted that, for different frequencies, $t_{0, \omega}, t_{1, \omega}, t_{2, \omega}$ and $c_{\omega}$ are identified independently, which causes that the EPSD model of the seismic ground motion includes too many independent parameters. In order to reduce the number of the parameters, we assume that $t_{0, \omega}, t_{1, \omega}$, $t_{2, \omega}$ and $c_{\omega}$ are the power functions of $\omega$, as

$$
\begin{aligned}
t_{0, \omega} & =P_{0} \cdot Q_{0}{ }^{-R_{0} \cdot \omega} \\
t_{1, \omega} & =P_{1} \cdot Q_{1}{ }^{-R_{1} \cdot \omega} \\
t_{2, \omega} & =P_{2} \cdot Q_{2}{ }^{-R_{2}} \cdot \omega \\
c_{\omega} & =P_{c} \cdot Q_{c}{ }^{-R_{c} \cdot \omega}
\end{aligned}
$$

To obtain the reasonable values of the parameters in Eq. (39), the other seismic ground motions recorded at the SMART-1 array during Event 45 in the E-W direction are modeled. All the ground motions are aligned by the peaks of the cross correlation functions to eliminate the wave-passage effect [30]. The identified values of $A_{0}, \varepsilon, f_{a}, f_{b}, \xi_{g}$ and $\omega_{g}$ are shown in Table 1 . The Coefficients of variation of those parameters are in the range of $0.09-0.34$. The identified values of $t_{0, \omega}, t_{1, \omega}, t_{2, \omega}, c_{\omega}$ and the corresponding mean values of different frequencies are presented in Fig. 13. The parameters in Eq. (39) are identified by letting Eq. (39) approximate to the mean values of $t_{0, \omega}, t_{1, \omega}, t_{2, \omega}$ and $c_{\omega}$. Table 2 has presented the identified values of $t_{0, \omega}, t_{1, \omega}, t_{2, \omega}$ and $c_{\omega}$ by the leastsquares approximation. Fig. 14 shows the comparisons between the mean values and the fitted models of $t_{0, \omega}, t_{1, \omega}, t_{2, \omega}$ and $c_{\omega}$ respectively. The modeling results show that Eq. (39) is available to describe the intensity variation of different frequency components in the time domain. However, as shown in Fig. 13, the discreteness of the identified values of these parameters is significant. 
a).

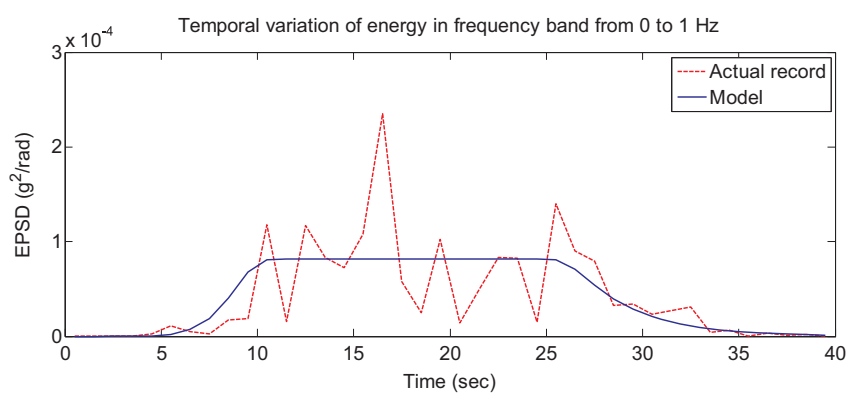

b).

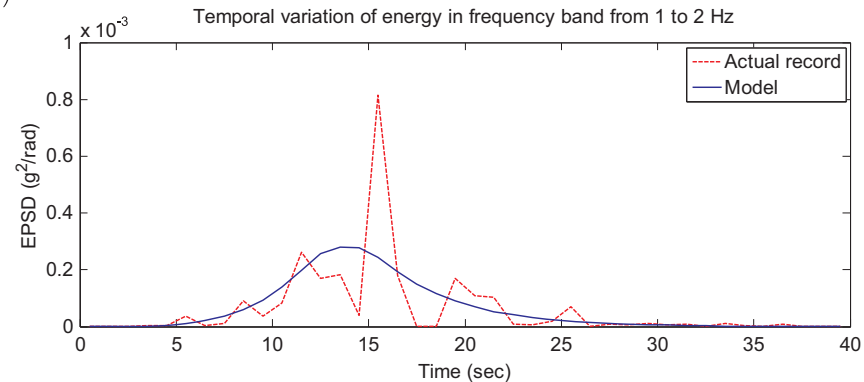

c).

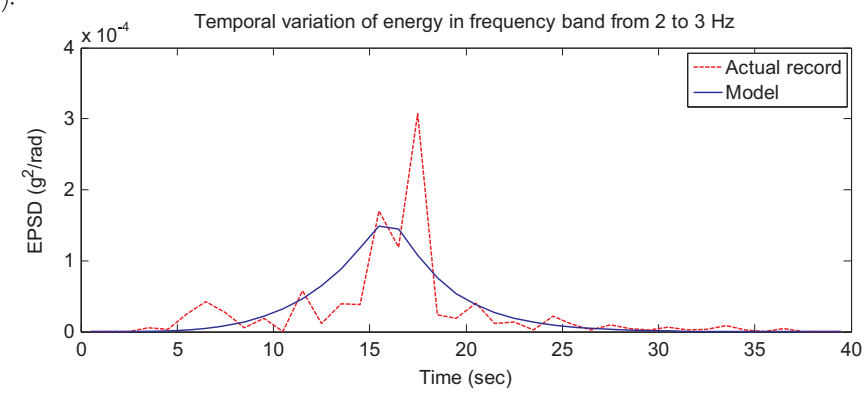

d).

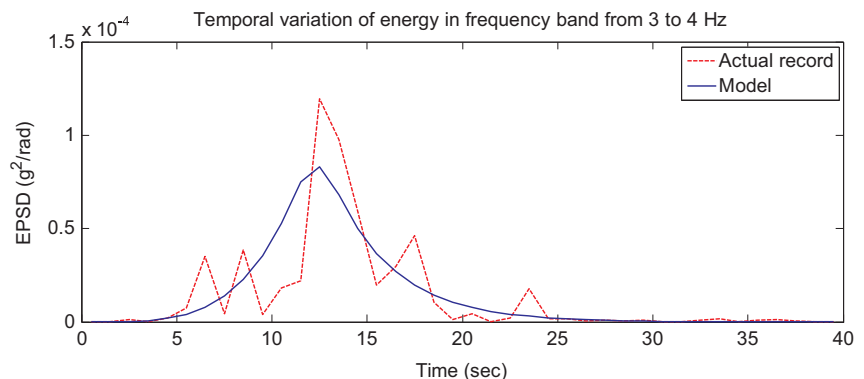

e).

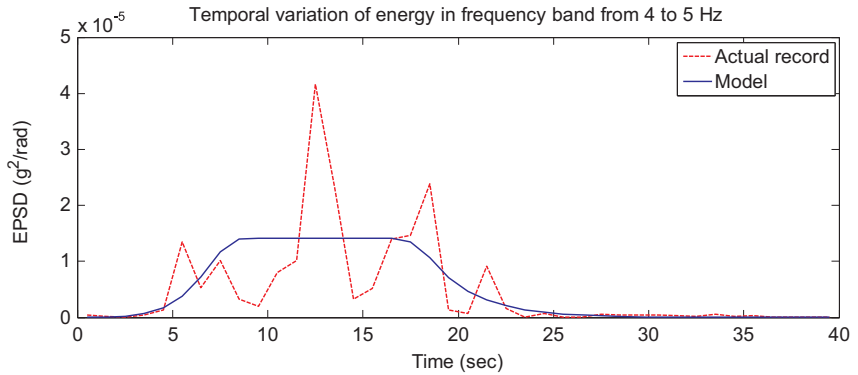

f).

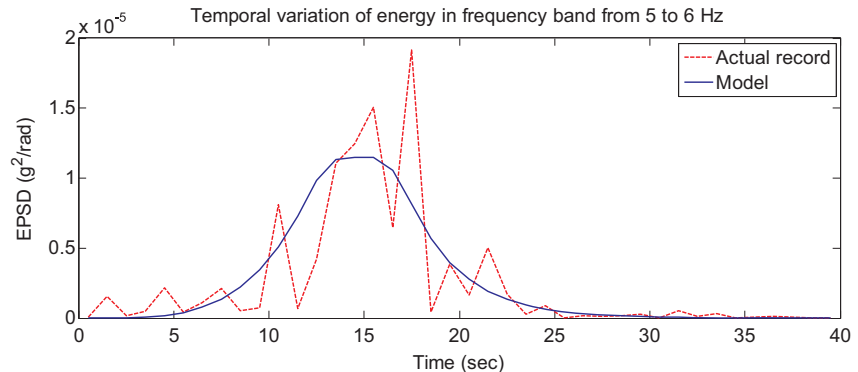

g).

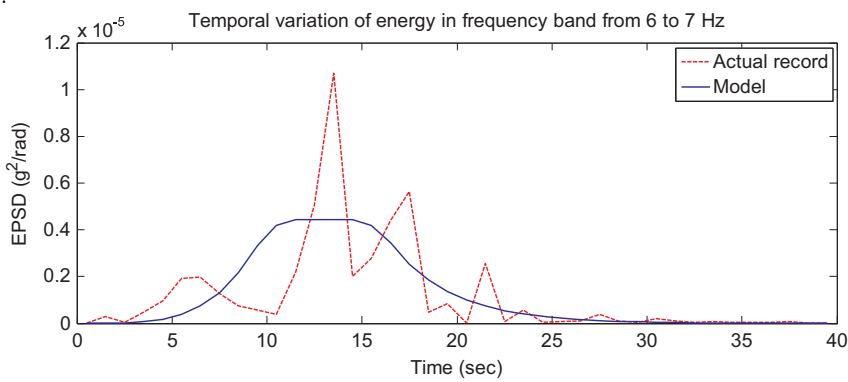

Fig. 8. Temporal variations of the energy of the actual ground motion record and fitted models in different frequency bands.

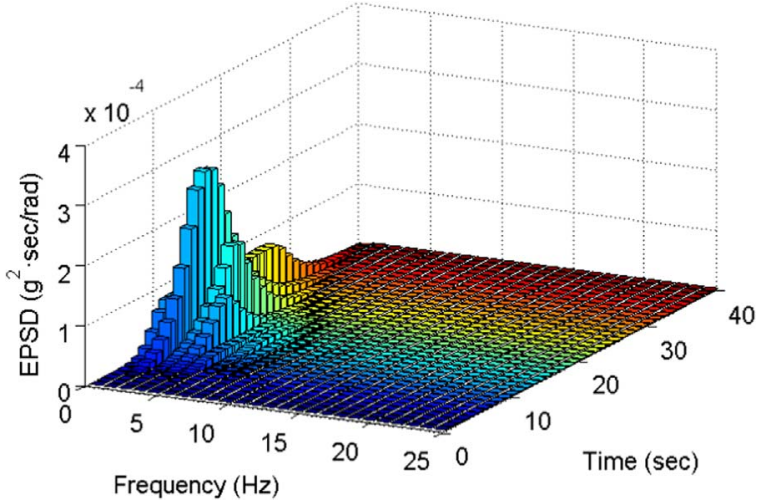

Fig. 9. Fitted EPSD model of the seismic ground motion presented in Fig. 3.

\section{Conclusions}

In this paper, a parametric evolutionary power spectrum model of fully nonstationary seismic ground motion is presented. This model consists of a frequency-domain energy distribution function and a series of normalized time-dependent envelope functions for different frequencies. The effects of the earthquake source and the local site are both considered in the modeling of the frequency-domain energy distribution. The normalized envelope functions are used to model the temporal energy variation of different frequency components of the seismic ground motion. The identification of the parameters in the EPSD model is divided into three steps. First, the actual EPSD of the seismic ground motion record is estimated via the generalized harmonic wavelets. Then, the amplitude parameter, $A_{0}$, and the parameters in $A_{s}(\omega)$ are identified by letting the frequency-domain energy distribution function of the EPSD model approximate to the actual frequency- 

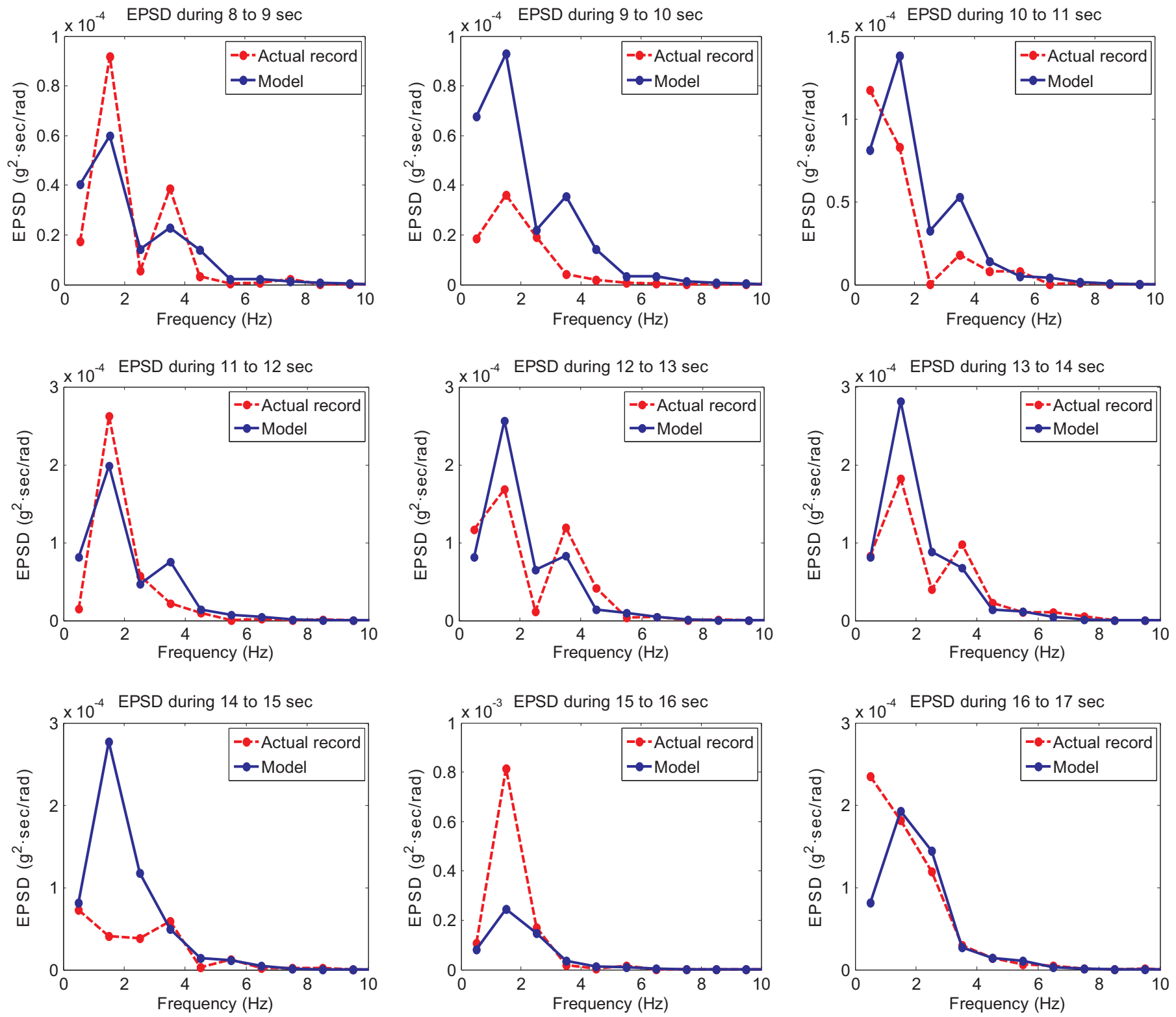

Fig. 10. The comparisons between the estimated EPSD of the actual ground motion record and the fitted model during 8-17 s.

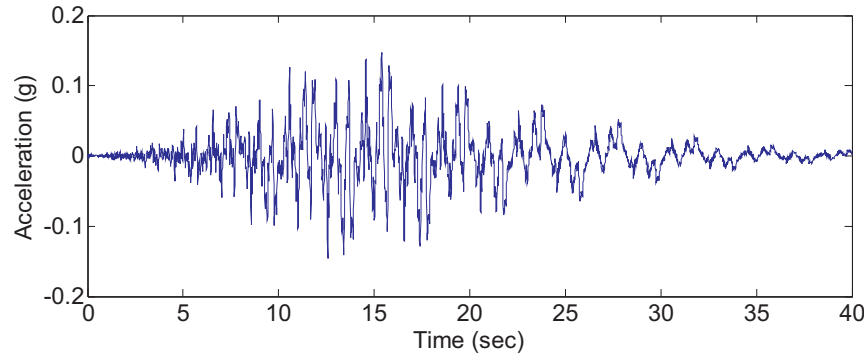

Fig. 11. Simulated ground motion acceleration using the fitted EPSD model.

domain energy distribution of the ground motion record. The actual frequency-domain energy distribution could be calculated by the estimated EPSD of the ground motion record. Further, for different frequencies, the parameters in the normalized envelope functions are identified by letting the corresponding normalized cumulative energy function of the EPSD model approximate to the actual normalized cumulative energy curves of the realistic ground motion. These actual normalized cumulative energy curves can also be calculated by the

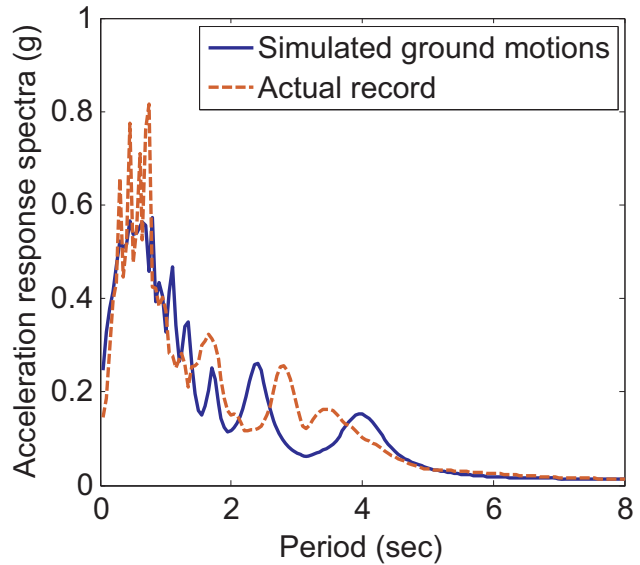

Fig. 12. The comparison between the response spectrum of the actual ground motion record and the mean response spectrum of 300 simulated ground motion samples by using the spectral representation method. 
a).

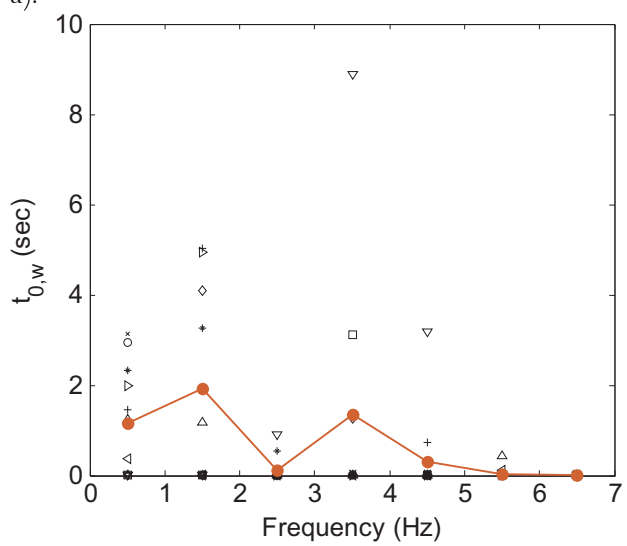

b).

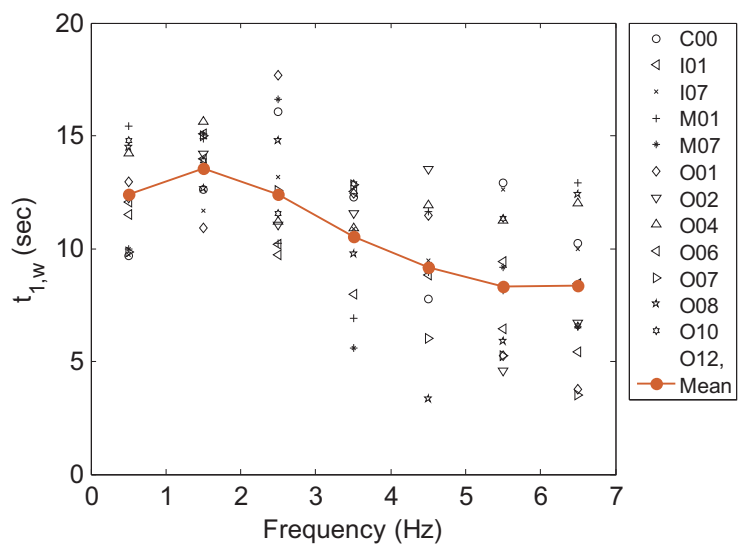

c).

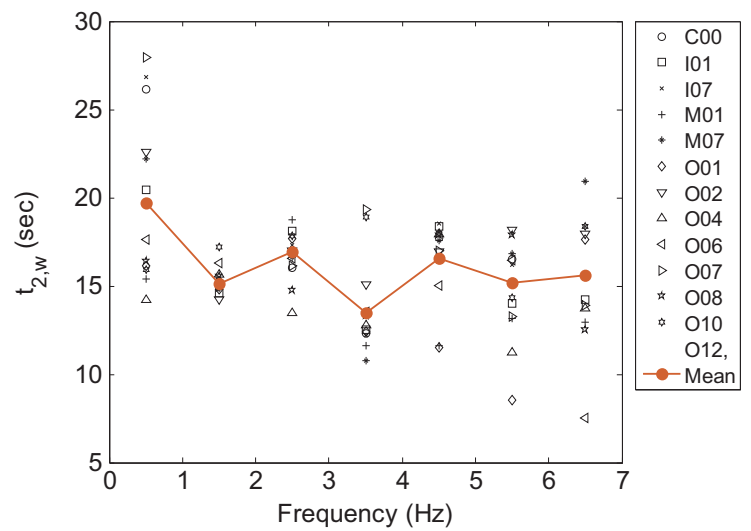

d).

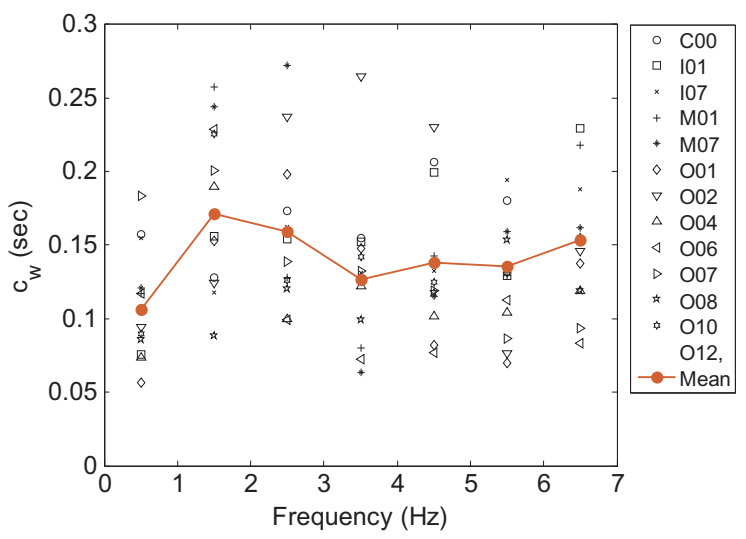

Fig. 13. Identified values of $t_{0, \omega}, t_{1, \omega}, t_{2, \omega}, c_{\omega}$ at different stations and the corresponding mean values.

a).

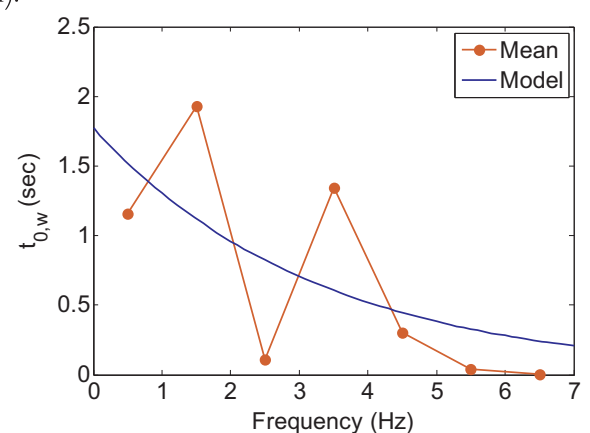

b).

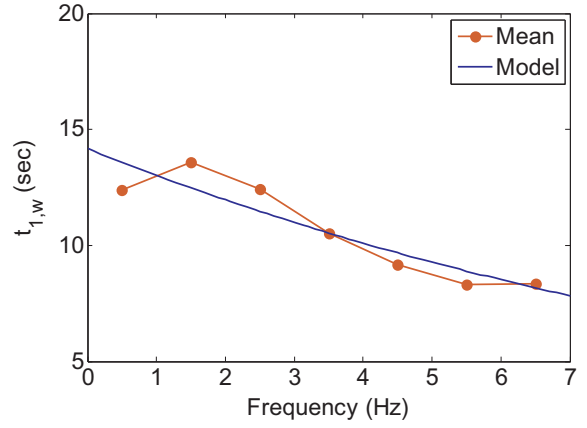

c).

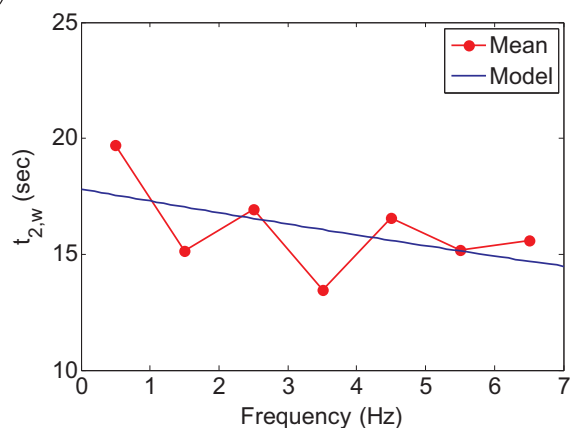

d).

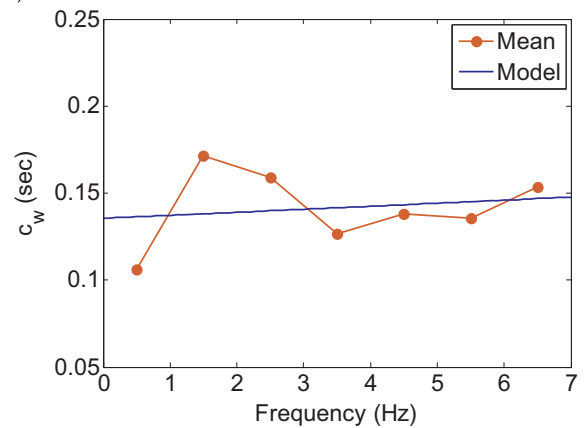

Fig. 14. Comparison between the mean values of $t_{0, \omega}$, $t_{1, \omega}, t_{2, \omega}, c_{\omega}$ and the fitted models. 
estimated EPSD of the ground motion record.

The modeling results show that the proposed EPSD model is available and effective to describe the temporal and spectral nonstationary characteristics of the realistic seismic ground motion. By the spectral representation method, this EPSD model could be used to synthesize artificial nonstationary earthquake time histories.

It should be noted that the parameters in the amplitude envelope functions are identified independently for different frequencies. At present, a type of deterministic power functional model is proposed to reflect the general tendency of these parameters varying with frequency. In the future, a stochastic model will be presented to describe the variation of the statistical characteristics of these parameters for different frequencies. In this way, the spectral nonstationarity of the seismic ground motion will be modeled more accurately in a statistical sense, and the proposed EPSD model will be more effective for engineering application.

\section{Acknowledgments}

This research was supported by National Natural Science Foundation of China (51408526 and 51308487), Natural Science Foundation of Hebei Province (E2015203274) and National Basic Research Program of China (2013CB834100).

\section{References}

[1] Rezaeian S, Der Kiureghian A. A stochastic ground motion model with separable temporal and spectral nonstationarities. Earthq Eng Struct Dyn 2008;37(13):1565-84.

[2] Yeh CH, Wen YK. Modeling of non-stationary ground motion and analysis of inelastic structural response. Struct Saf 1990;8:281-98.

[3] Wang J, Fan L, Qian S, Zhou J. Simulations of non-stationary frequency content and its importance to seismic assessment of structures. Earthq Eng Struct Dyn 2002;31:953-1005.

[4] Beck JL, Papadimitriou C. Moving resonance in nonlinear response to fully nonstationary stochastic ground motion. Probabilistic Eng Mech 1993;8(3):157-67.

[5] Conte JP, Peng BF. Fully nonstationary analytical earthquake ground-motion model. J Eng Mech 1997;123(1):15-24.

[6] Wang J, Fan L, Qian S, Zhou J. Simulations of nonstationary frequency content and its importance to seismic assessment of structures. Earthq Eng Struct Dyn 2002;31(4):993-1005.

[7] Housner GW. Characteristics of strong-motion earthquakes. Bull Seismol Soc Am 1947;37(1):19-27.

[8] Bolotin VV. Statistical theory of the aseismic design of structures. In: Proceedings of the 2nd world conference on earthquake engineering, Tokyo and Kyoto, Japan, Vol. 2 ; 1960. p. 1365-74.

[9] Jennings PC, Housner GW, Tsai NC. Simulated earthquake motions [Tech.rep.].
Pasadena, California, USA: Earthquake Engineering Research Laboratory, California Institute of Technology; 1968.

[10] Amin M, Ang AH-S. Non-stationary stochastic models of earthquake motion. J Eng Mech Div ASCE 1968;94:559-83.

[11] Saragoni GR, Hart GC. Simulation of artificial earthquakes. Earthq Eng Struct Dyn 1974;2:249-67.

[12] Priestley MB. Evolutionary spectra and nonstationary processes. J R Stat Soc Ser A 1965;27:204-37.

[13] Liu SC. Evolutionary power spectral density of strong-motion earthquakes. Bull Seismol Soc Am 1970;60(3):891-900.

[14] Lin YK, Yong Y. Evolutionary Kanai-Tajimi earthquake model. J Eng Mech 1987;113(8):1119-37.

[15] Kameda H, Sugito M, Asamura T. Simulated earthquake motions scaled for mag nitude, distance and local soil conditions. In: Proceedings of the 7th world conference on earthquake engineering, Istanbul, Turkey,Vol. 2; 1980. p. 295-302.

[16] Der Kiureghian A, Crempien-Laborie JE. An evolution model for earthquake ground motion. Struct Saf 1989;6:235-46.

[17] Crempien-Laborie JE, Orosco L. An evolution ground motion model for earthquake analysis of structures in zones with little history. Soil Dyn Earthq Eng 2000;20:373-9.

[18] Yamamoto Y, Baker JW. Stochastic model for earthquake ground motion using wavelet packets. Bull Seismol Soc Am 2013;103(6):3044-56.

[19] Shinozuka M, Jan CM. Digital simulation of random process and its applications. J Sound Vib 1972;25(1):111-28.

[20] Shinozuka M, Deodatis G. Simulation of stochastic processes by spectral representation. Appl Mech Rev 1991;44(4):191-204.

[21] Deodatis G. Non-stationary stochastic vector processes: seismic ground motion application. Probabilistic Eng Mech 1996;11(3):149-67.

[22] Liang J, Chaudhuri SR, Shinozuka M. Simulation of nonstationary stochastic processes by spectral representation. J Eng Mech 2007;133(6):616-27.

[23] Cacciola P, Deodatis G. A method for generating fully non-stationary and spectrum compatible ground motion vector processes. Soil Dyn Earthq Eng 2011;31:351-60.

[24] Spanos PD, Failla G. Evolutionary spectra estimation using wavelets. J Eng Mech ASCE 2004;130:952-60.

[25] Spanos PD, Tezcan J, Tratskas P. Stochastic processes evolutionary spectrum estimation via harmonic wavelets. Comput Methods Appl Mech Eng 2005;194:1367-83.

[26] Schillinger D, Papadopoulos V. Accurate estimation of evolutionary power spectra for strongly narrow-band random fields. Comput Methods Appl Mech Eng 2010;199:947-60.

[27] Newland DE. Harmonic wavelet analysis. Proc R Soc A: Math Phys Eng Sci 1993;443:203-5.

[28] Newland DE. Harmonic and music wavelets. Proc R Soc A: Math, Phys Eng Sci 1994:444:605-20.

[29] Boore DM. Simulation of ground motion using the stochastic method. Pure Appl Geophys 2003;160:635-76.

[30] Zerva A. Spatial variation of seismic ground motions: modeling and engineering applications. New York: CRC Press; 2009.

[31] Aki K. Scaling law of seismic spectrum. J Geophys Res 1967;72(4):1217-31.

[32] Atkinson GM, Boore DM. Ground motion relations for eastern North America. Bull Seismol Soc Am 1995;85:17-30.

[33] Haddon R. Earthquake source spectra in eastern North America. Bull Seismol Soc Am 1996;86:1300-13.

[34] Atkinson GM, Silva W. Stochastic modeling of California ground motions. Bull Seismol Soc Am 2000;90:255-74. 